\title{
Conditioned pain modulation in drug-naïve patients with de novo Parkinson's disease
}

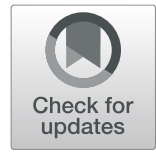

Wiebke Grashorn ${ }^{1 \dagger}$, Odette Fründt ${ }^{1 \dagger}$, Carsten Buhmann ${ }^{1}$, Nathalie Wrobel ${ }^{3}$, Katharina Schmidt ${ }^{2 *}$ and Ulrike Bingel ${ }^{2,4}$

\begin{abstract}
Background: Pain is highly prevalent in patients with Parkinson's disease (PD), but underlying pathophysiological mechanisms are largely unclear. In many chronic pain syndromes deficits in endogenous pain inhibition have been detected that can be assessed using conditioned pain modulation paradigms. Previous studies employing this approach in medicated PD patients did not find abnormal pain inhibition. However, these results might have been confounded by residual dopaminergic medication.

Methods: An established conditioned pain modulation paradigm was used in 17 drug-naïve de novo PD patients and 17 healthy age and gender-matched controls. We tested i) whether conditioned pain modulation responses differed between the patient and control group and ii) whether pain inhibition differed between PD subtypes.

Results: PD patients and healthy controls did not differ in their conditioned pain modulation responses. Furthermore, there were no significant differences in CPM responses depending on the PD subtype. However, at a descriptive level, tremor-dominant patients showed a tendency for better descending pain inhibition compared to akinetic-rigid and mixed type patients.

Conclusions: In this first study investigating conditioned pain modulation in de novo PD patients, we found no additional impairment in descending pain modulation besides the known age-related decline. Our findings indicate that mechanisms other than an impaired descending inhibition contribute to high pain prevalence rates in PD and suggest that mechanisms underlying pain may differ between PD subtypes.
\end{abstract}

Keywords: CPM, Descending inhibition, Neurodegeneration, Dopamine

\section{Introduction}

Pain is a highly prevalent symptom in patients with Parkinson's disease (PD) and affects up to $90 \%$ of the patients $[3,8]$. It significantly impairs patients' quality of life $[42,44]$ and has been reported to precede motor symptoms [50]. Charcot already described pain in PD in 1878 [18], yet only little is known about its underlying pathophysiological mechanisms.

The susceptibility to acute and chronic pain is supposed to depend on the balance of activity in ascending and descending pain pathways [33, 38, 51]. The descending pain control system can modulate pain by either inhibiting or facilitating nociceptive processing $[33,38]$. Because parts of the descending pain inhibitory

\footnotetext{
* Correspondence: Katharina.schmidt@uk-essen.de

tWiebke Grashorn and Odette Fründt contributed equally to this work.

${ }^{2}$ Department of Neurology, University Hospital Essen, University

Duisburg-Essen, Hufelandstrasse 55, 45147 Essen, Germany

Full list of author information is available at the end of the article
}

system involve dopaminergic pathways (i.e., in the rostral agranular insular cortex and dorsal horn neurons) [4, 9], dysregulation of dopaminergic transmission might contribute to altered pain processing in PD.

Conditioned pain modulation (CPM) paradigms, in which pain intensity ratings of noxious test stimuli are obtained in the presence and absence of a concomitantly presented conditioning stimulus to a remote body part [53], represent a well-established way to study descending pain inhibition in humans. Positive CPM responses (i.e., reduced pain intensity ratings under concurrent stimulation) are indicative of endogenous analgesia and are mediated by spino-bulbo-spinal reflexes [32], which are controlled by higher cortical brain areas [34, 41, 46].

To date, there are only few studies investigating CPM responses in patients with idiopathic Parkinson reporting no significant differences in CPM responses in medicated PD patients as compared to healthy controls [22,

(c) The Author(s). 2019 Open Access This article is distributed under the terms of the Creative Commons Attribution 4.0 International License (http://creativecommons.org/licenses/by/4.0/), which permits unrestricted use, distribution, and 
23, 35]. Furthermore, over-night withdrawal of medication had no effect on CPM responses in PD patients [22, 23]. However, this washout phase might have been too short to sufficiently eliminate the dopaminergic medication. Although the plasma half-life period of dopamine agonists is relatively short (usually several hours) [7] and that of levodopa is generally estimated as 0.7 to $1.4 \mathrm{~h}$ [11], the latter can last up to 7.9 days [15]. The residual dopaminergic concentration might therefore still have induced antinociceptive effects [4] and could have normalized a reduced CPM response in PD patients. A recent study in patients with restless leg syndrome suggested that antinociceptive/analgesic effects of dopamine are concentration-dependent [4]. Low dopaminergic concentrations induced antinociceptive effects via dopaminergic D2 receptors, whereas higher levels had pro-nociceptive effects based on the activation of D1 receptors $[4,40]$. Dopamine could therefore either increase or decrease CPM responses in PD patients depending on its concentration and low concentrations might have led to decreased pain during the insufficient washout phase.

In order to avoid this and other treatment-related confounds, we studied CPM responses using an established paradigm $[23,24,46]$ in drug-naïve de novo $\mathrm{PD}$ patients. Specifically, we investigated whether CPM responses differed between de novo PD patients and healthy controls. On the assumption that distinct patterns of neurodegeneration underlying different PD subtypes [39] might affect pain modulation differentially, we also assessed the influence of the PD subtype on CPM responses, which has not been assessed in previous studies.

\section{Methods}

\section{Participants}

The study was conducted between 2011 and 2013 at the Department of Neurology at the University Medical Center Hamburg.

De novo PD patients were recruited from our movement disorders outpatient clinic (head: Prof. Carsten Buhmann) and had to fulfill the following inclusion criteria: (1) diagnosis of idiopathic PD according to the criteria of the UK PD Society Brain Bank, (2) Hoehn \& Yahr scale $\leq$ stage III [27], (3) naïve to dopaminergic medication ("de novo" patients), (4) age between 40 and 90 years, (5) no severe cognitive impairment (Parkinson Neuropsychometric Dementia Assessment (PANDA; [28]) instrument 215 ), (6) no manifest depression or anxiety, (7) no acute pain or analgesic medication during the last $24 \mathrm{~h},(8)$ no history of chronic pain disorders e.g. rheumatoid arthritis (PD specific chronic pain [16] was allowed), (9) no regular use of prescription analgesics, tranquilizers, antidepressants, pain modulating anticonvulsants (e.g. gabapentin or pregabaline), and (9) no pregnancy.

Healthy controls matched for age (+/ -3 years) and gender were recruited locally by announcement and had to fulfill the same inclusion criteria except for (1) and (2). This matched control group of the same size was used to calculate group comparisons to reveal potential abnormalities of measured parameters in the de novo group.

Out of the initially recruited sample size of 25 patients and 24 healthy controls, eight patients and seven healthy controls had to be excluded due to not fulfilling the inclusion criteria mentioned above or withdrawal from the study during the exposure to ice water. In detail, one patient decided to withdraw from the study, six had started dopaminergic medication at the day the study was performed, and one patient and seven healthy controls withdrew during the exposure to ice water. Only participants who finished the study were included into data analysis.

\section{Experimental protocol}

In this study, we used an established CPM paradigm $[23,24,46]$, which combines painful heat stimuli as test stimuli (TS) with a cold pressor task as the conditioning stimulus (CS). Patients were informed about the study, its purpose, and the study protocol using a standardized protocol after a regular visit in our outpatient clinic. After answering their questions, inclusion criteria were checked and written information material was handed out.

In brief, the experimental procedures included an introductory session, which consisted of a clinical interview to check again all aforementioned inclusion criteria and re-evaluate the PD diagnosis, the assessment of Hoehn and Yahr stage, Unified Parkinson's Disease Rating Scale and PD subtype, filling in of questionnaires (see Assessment of anxiety and depression), and the calibration of stimulus intensities (see Instructions and calibration procedure). This was followed by the a priori assessment of expectation ratings regarding possible changes of pain intensities during the application of the cold pressor task. Finally, the actual CPM paradigm was performed. The paradigm consisted of three blocks, in which six test stimuli each were applied to the right volar forearm. Pain ratings to these stimuli were obtained before (=block I), during (=block II) and after (=block III) a cold pressor task that was applied to the contralateral left leg during the second block. The experimental protocol is summarized in Fig. 1. All experimental parts were performed on the same day in one single session usually a few days (weeks) after patients had been informed about the study in our outpatient clinic. 


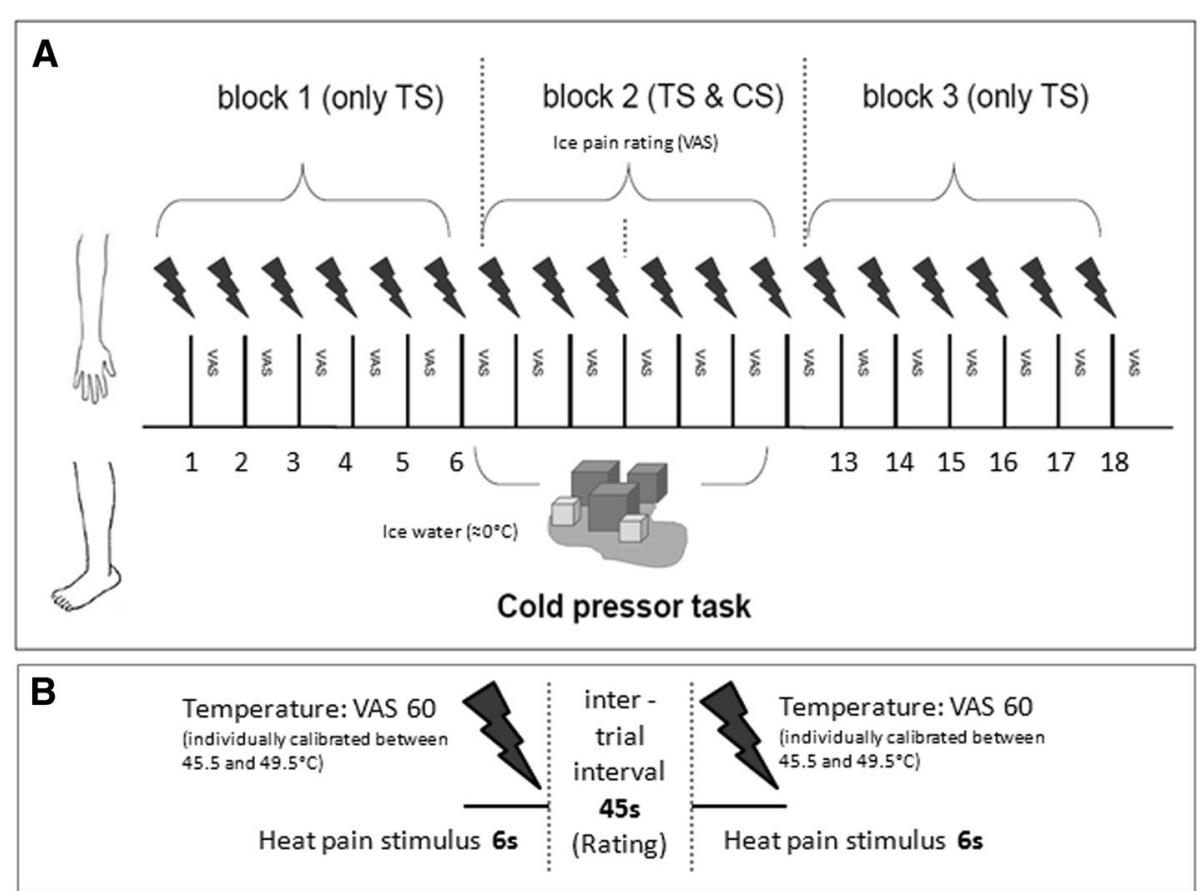

Fig. 1 Experimental sequences of the CPM paradigm used in this study. (a whole experiment, $\mathbf{b}$ temporal components of trials). During block 1 and 3, the test stimulus (TS) was applied alone, whereas during block 2, the TS and a conditioning stimulus (= cold pressor task using ice water; CS) were applied concurrently. Subjects had to rate the pain intensity of TS and CS on a visual analogue scale (VAS)

\section{Instructions and calibration procedure}

All participants were instructed using a standardized protocol. Participants were told that the purpose of the study was to characterize possible differences in the perception of two simultaneously applied painful stimuli comparing PD patients with healthy participants of the same age. First, participants were informed about the sequence of experimental procedures. These general instructions were followed by a clinical interview (both groups) checking all inclusion criteria. In the PD group, an experienced clinician re-evaluated the PD diagnosis and assessed the individual Hoehn and Yahr stage [26], Unified Parkinson's Disease Rating Scale (UPDRS [14]) score (total and motor score (part III)), and PD subtype. The PANDA [28] tested for cognitive impairment. Subjects with scores $<15$ were excluded to ensure a sufficient task comprehension and compliance. PD subtypes were classified clinically according to the German AWMF Guidelines (www.awmf.org) as tremor-dominant $(n=9)$, akinetic-rigid $(n=5)$ or mixed $(n=3)$ depending on the predominant motor symptom (tremor, bradykinesia/rigidity or an equal manifestation of both) that had to be predominant at symptom onset and over the course of disease. Both groups completed the HADS [56] as depression and anxiety can modulate pain perception $[1,10]$ and acute pain was assessed asking the patients for any pain they might have experienced during the $24 \mathrm{~h}$ prior to the experiment (in case subjects experienced pain during $24 \mathrm{~h}$ prior to the experiment subjects were excluded from the study). If subjects fulfilled the inclusion criteria (see Participants) a calibration procedure was performed to determine the individual temperatures corresponding to a pain level of $50-60$ on a $0-100$ visual analogue scale [VAS, endpoints $0-100]$. To this end, we applied 10 stimuli á $6 \mathrm{~s}$ each with different intensities ranging from $45.5-49.5^{\circ} \mathrm{C}$ in a pseudo-randomized order to the right volar forearm, every temperature was presented once. Participants were asked to rate the intensity of each stimulus on a VAS, which was presented on a computer screen in front of the subjects and ranged from $0=$ "no sensation" to $100=$ "most intense pain imaginable". Two vertical white lines represented the two endpoints 0 and 100 of the VAS, a third white line was set at 25 labeled as "pain threshold" to assess non-painful sensations, which might occur during the cold pressor task as a result of an effective pain inhibition. Subjects indicated the pain intensity of each heat pain stimulus by moving a red bar between the two endpoints using two buttons of a computer mouse. Participants did have as much time as they needed to provide their ratings, the experiment continued afterwards. The maximum stimulation temperature was restricted to $49.5^{\circ} \mathrm{C}$ in order to avoid any tissue damage. The calibration procedure ensured that all participants perceived the phasic heat pain stimuli (= test stimuli, TS) as comparably painful (VAS 50-60). 
The application of the thermal stimuli, the presentation of the VAS and the recording of behavioral data was performed using the software "Presentation" (www.neurobs.com).

\section{Test stimulus}

We used phasic heat pain stimuli as test stimuli (TS). The test stimuli were applied to the right volar forearm $(\sim 10$ $\mathrm{cm}$ proximally from the wrist) using a $30 \times 30 \mathrm{~mm}$ PeltierThermode (TSAII, Medoc, Israel). Each stimulus had a duration of $6 \mathrm{~s}$ (baseline temperature $35^{\circ} \mathrm{C}$, ramp up and down $10^{\circ} \mathrm{C} /$ second, destination temperature individually calibrated between 45.5 and $49.5^{\circ} \mathrm{C}$, interstimulus-interval $\sim 45 \mathrm{~s}$ ). Pain ratings on the VAS were obtained immediately after each stimulus. A total of 18 test stimuli were applied. The first (=block $I$, stimulus 1 to 6 ) and the last six stimuli (= block III, stimulus 13 to 18 ) were applied without any other concomitant procedures. During the application of test stimuli 7 to 12 (=block II), the conditioning stimulus was applied.

\section{Conditioning stimulus}

A cold pressor task was used as the conditioning stimulus (CS). After completion of the first block of 6 heat pain stimuli (block I), a message on the computer screen prompted the participants to immerse their left foot into a bath with ice water $\left(\sim 0^{\circ} \mathrm{C}\right)$. The intensity of the conditioning stimulus was rated once in the middle of the cold pressor task (= after TS 9, block II) using a VAS presented on a computer screen with the same endpoint labels $0=$ "no sensation" and $100=$ "most intense pain imaginable" and a third white line set at 25 labeled as "pain threshold". At the end of block II, another message on the computer screen instructed the participants to take their foot out of the ice water. After taking their foot out of the ice water, participants positioned their foot in a towel on the floor next to the tub with ice water. Prior to the experiment, subjects were asked to focus their attention on the heat stimuli applied to the arm while having their foot immersed into the ice water and it was pointed out again that they could withdraw from the experiment at any time by telling the supervising experimenter. Finally, heat pain stimuli 13 to 18 (block III) were applied without concomitant painful stimulation to the foot.

\section{Assessment of individual expectation}

Many cognitive and affective processes could influence CPM responses. However, expectations which are known to modulate pain have previously been suggested to affect CPM responses [12, 21, 31, 36]. Following the calibration procedure, prior to the actual experiment, patients were presented the following question on the computer screen: "How do you expect the pain applied to your arm to change while you have your foot immersed into the ice water?" Participants were asked to indicate their expectations on a computerized VAS with the verbal anchors $1=$ "no sensation" (=pain at the arm would be completely abolished during the cold pressor task), $0=$ "no change" (= no change of heat pain at the arm during the cold pressor task), and $1=$ "maximum pain" (=pain applied to the arm would get worse during the cold pressor task). Two vertical white lines represented the two endpoints - 1 ("no sensation") and 1 ("maximum pain") of the VAS, a third white line was set at 0 labeled as "no change". Subjects indicated their expectation by moving a red bar between the two endpoints using two buttons of a computer mouse. Participants did have as much time as they needed to provide their ratings. As in previous studies, no specific suggestions regarding the direction of possible changes were divulged [12].

\section{Assessment of anxiety and depression}

The Hospital Anxiety and Depression Scale (HADS) [56] is a self-report questionnaire to assess anxiety and depression with 7 items per subscale. Each item is scored from 0 to 3 points so that scores of 21 points for each subscale depression and anxiety can be reached. Higher scores indicate higher symptom severity. Both subscales have been validated to have good sensitivity and specificity [5].

\section{Data analysis}

Data analysis was performed using IBM SPSS 20.0. There was no missing data. Non-parametric tests were used in case the assumptions of variance homogeneity (Levene's test) and normal distribution (Kolmogorov-Smirnov test) were violated. For between-group comparisons between patients and controls, we used two-sample t-tests and non-parametric Mann-Whitney U tests.

CPM responses were calculated as the difference between mean pain ratings before and after the CS and mean pain ratings during the cold pressor task (CPM response $=($ mean pain ratings of blocks $(1+3))-($ mean pain rating of block 2)) as described in previous studies $[23,24,46]$. A positive CPM response indicates a reduction in pain perception during the cold pressor task and therefore signifies analgesia symbolizing effective descending pain inhibition mechanisms, whereas a negative CPM response shows an increase of pain ratings in block II.

As different methods to calculate CPM responses are described (for review see [43, 54]), we also analyzed descending pain inhibition mechanisms in our participants using two other methods:

- block 1 - block 2: possible differences between patients and controls regarding mean pain ratings of test stimuli before (=block 1$)$ and during CS (= block 2) 
- block 1 mean (stimulus1,2,3) - block 2 mean (stimulus1,2,3): possible differences between patients and controls regarding mean pain ratings of the first 3 test stimuli (mean of block 1 stimulus 1,2,3) and the first 3 stimuli of block 2 (mean of block 2 stimulus $1,2,3)$.

This data is presented in the Additional file 1.

To test for group-specific CPM responses in healthy controls and patients, separate one sample t-tests on CPM responses were performed for the PD group and the control group. Kruskal-Wallis tests were used for PD subtype analyses. Correlations were calculated using Pearson's ( $r-$ value) or non-parametric Spearman's coefficients (rhovalue). $P$-values $<0.05$ were considered as statistically significant. Test results were corrected for multiple comparisons using Bonferroni correction.

\section{Results}

\section{Clinical and neuropsychiatric assessment}

Seventeen de novo PD patients (mean 61.9 years +/standard deviation (SD) 9.8, range 42-75) and 17 healthy controls $(61.7+/-9.8$, range $45-75)$ matched in age $(t(32)=-0.070, p=0.945)$ and gender (each group: 10 male, 7 female) were included in the study. PD patient characteristics are given in Table 1. All group comparisons between patients and controls are given in Table 2. Mean PANDA scores differed significantly between both groups with lower PANDA scores in the patient group. Mann-Whitney U tests revealed a significant difference between the groups in the HADS depression subscore with higher scores in the patient group. HADS anxiety scores were comparable between both groups.

\section{Experimental parameters and expectation ratings}

Stimulation temperatures and mean pain ratings in block 1 did not differ significantly between groups (see Table 2). Mean cold pain ratings only differed by trend $(p=0.053)$ with slightly higher pain ratings in controls. Interestingly, expectation ratings differed between both groups. De novo patients expected the heat pain to be similar or slightly increased under concurrent ice stimulation, whereas controls expected it to be reduced. There was no significant correlation of expectation with any of the clinical scores (PANDA, HADS_A, HADS $D$, UPDRS total and motor score and symptom duration) or with CPM responses when pooling across groups and when analyzing these correlations for each group separately.

\section{CPM responses}

CPM responses did not differ between controls and patients $(p=0.545$, see Table 2 and Fig. 2). Analyses of group-specific CPM responses using one sample t-tests revealed that neither patients $(1.7+/-6.7 ; t(16)=1.058, p=$ $0.306)$ nor controls $(3.1+/-6.4 ; t(16)=1.989, p=0.064)$ showed a significant CPM response.

CPM responses of the alternative calculation methods (a) block 1 - block 2 and b) block 1 mean (stimulus1,2, 3) - block 2 mean (stimulus1,2,3)) are presented in the Additional file 1.

\section{CPM responses and PD subtypes}

Kruskal-Wallis tests revealed no significant differences between the 3 subtypes with respect to age, UPDRS scores, PANDA scores, the HADS subscales for anxiety and depression, stimulation temperature, mean pain intensity rating in block 1 and cold pain ratings. Tremor-dominant patients showed greater CPM responses $(n=9: 3.9+/-6.4$

Table 1 Clinical characteristics of de novo Parkinson patients

\begin{tabular}{|c|c|}
\hline Parameters & $\begin{array}{l}\text { Patient characteristics }(n=17) \\
\text { mean }+/- \text { standard deviation [Min - Max] }\end{array}$ \\
\hline Age & 61.9 years $+/-9.8[42-75$ years $]$ \\
\hline Gender & 10 male, 7 female \\
\hline Handedness & 16 right-handed, 1 left-handed \\
\hline Hoehn \& Yahr Scale (H \& Y) & $\begin{array}{l}\text { - H \& Y stage I: } 4 \text { patients } \\
\text { - H \& Y stage II/ II.5: } 12 \text { patients } \\
\text { - H \& Y stage III: } 1 \text { patients }\end{array}$ \\
\hline PD subtype & $\begin{array}{l}\text { - akinetic-rigid: } 5 \text { patients } \\
\text { - tremor-dominant: } 9 \text { patients } \\
\text { - mixed type: } 3 \text { patients }\end{array}$ \\
\hline Clinically most affected half of the body & $\begin{array}{l}\text { - right: } 4 \text { patients } \\
\text { - left: } 13 \text { patients }\end{array}$ \\
\hline Disease duration (months since first time occurrence of symptoms prior to study) & 18 months +/- SD 15.8 [4-60 months] \\
\hline Unified Parkinson's Disease Rating Scale (UPDRS) - total score & $30.4+/-11.6[15-48]$ \\
\hline Unified Parkinson's Disease Rating Scale (UPDRS) - motor score & $21.2+/-8.3[9-33]$ \\
\hline
\end{tabular}


Table 2 Main results for healthy subjects and de novo Parkinson patients (PD) in group comparison

\begin{tabular}{|c|c|c|c|}
\hline $\begin{array}{l}\text { Parameters } \\
\text { (mean, standard deviation, [Min-Max]) }\end{array}$ & $\begin{array}{l}\text { Healthy subjects } \\
(n=17)\end{array}$ & $\begin{array}{l}\text { De novo Parkinson patients } \\
(n=17)\end{array}$ & $\begin{array}{l}\text { Group comparison Healthy subjects vs. De novo } \\
(p \text {-value) }\end{array}$ \\
\hline Age & $61.7+/-9.8[45-75]$ & $61.9+/-9.8[42-75]$ & $\mathrm{t}(32)=-0.070, p=0.945$ \\
\hline Gender & 10 male, 7 female & 10 male, 7 female & - \\
\hline PANDA Score & $24.6+/-$ SD $3.0[17-29]$ & $21.1+/-4.0[15-29]$ & $\mathrm{t}(32)=2.848, p=0.008$ \\
\hline HADS subscale depression & $1.3+/-1.3[0-4]$ & $3.5+/-2.5[0-9]$ & $\mathrm{U}=227.5, \mathrm{z}=2.901, p=0.003$ \\
\hline HADS subscale anxiety & $2.2+/-2.1[0-6]$ & $4.5+/-4.3[0-14]$ & $U=187.5, z=1.498, p=0.140$ \\
\hline Mean stimulation temperature & $48.2+/-0.8[47.0-49.5]$ & $47.9+/-0.9[45.5-49.0]$ & $U=80.5, z=-0.739, p=0.478$ \\
\hline $\begin{array}{l}\text { Mean pain intensity } \\
\text { Block } 1 \text { [VAS 0-100] }\end{array}$ & $55.9+/-8.8[38.7-67.0]$ & $60.8+/-12.1[37.8-86.8]$ & $\mathrm{t}(32)=-1.380, p=0.177$ \\
\hline Cold pain rating Block 2 [VAS 0-100] & $74.8+/-18.3[29-97]$ & $58.7+/-27.4[14-96]$ & $\mathrm{t}(27.874)=2.023, p=0.053^{*}$ \\
\hline $\begin{array}{l}\text { Expectation } \\
\text { Rating Day } 1[-1 \text { to }+1]\end{array}$ & $-0.2+/-0.4[-0.8-0.5]$ & $0.1+/-0.4[-0.7-0.9]$ & $\mathrm{t}(32)=-2.609, p=0.014$ \\
\hline CPM Magnitude & $3.1+/-6.4[-5.1-13.3]$ & $1.7+/-6.7[-9.8-13.9]$ & $t(32)=0.611, p=0.545$ \\
\hline
\end{tabular}

Significant results are marked in bold

Abbreviations: UPDRS Unified Parkinson's disease rating scale, HADS Hospital anxiety and depression score, PANDA Parkinson neuropsychometric dementia assessment

* $=P$-value and degree of freedom corrected for unequal variances

[-5.1-13.9]) compared to akinetic-rigid $(n=5: 0.4+/-8.1$ $[-9.8-10.8])$ and mixed subtype PD patients $(n=3$ : $-2.6+/-2.5$ [-4.3-0.3]; see Fig. 3). However, differences between groups did not reach statistical significance $(H(2)=2.287, p=0.319)$. One sample t-tests revealed no significant CPM response in none of the subtypes (all $p>0.100$ ).

\section{Correlations of CPM responses with other parameters}

There were no correlations between CPM responses and other parameters investigated in this study neither in patients nor in controls. As expected, in the patient group, UPDRS total and motor score correlation $(r=0.968, p<$ $0.001)$ as well as HADS_A and HADS_D correlation were very high $(r h o=0.801, p=0.000)$.

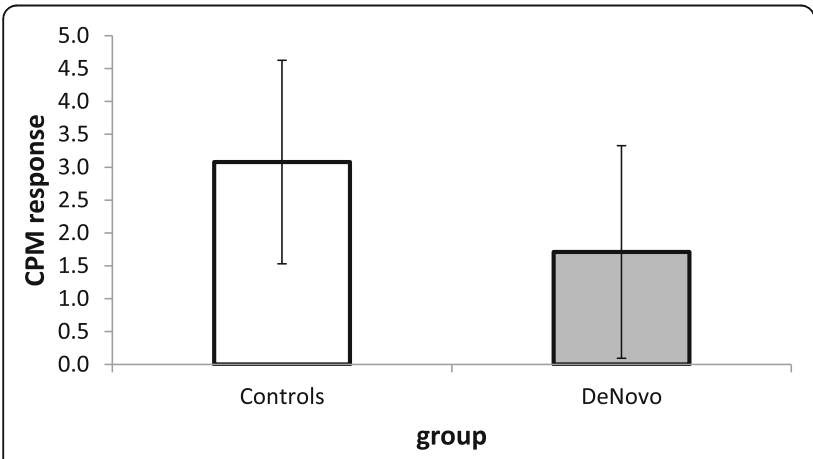

Fig. 2 Conditioned pain modulation magnitudes are shown for healthy controls and de novo PD patients. Means and standard error of means are presented. There were no group differences between de novo patients (gray bar) and controls (white bar)

\section{Discussion}

In this study, we used a well-established CPM paradigm to investigate endogenous pain modulation in drug naïve de novo PD patients. Our results do not show any significant differences between CPM responses of de novo PD patients and healthy subjects matched for age and gender, indicating no additional impairment of descending pain inhibition in PD besides the known age-related decline in CPM responses $[13,24,31,52]$. In this study, we have chosen an established CPM paradigm that has been applied in previous studies [23, 24, 46]. However, several different methods to calculate CPM response are described [43, 54]. Here, using two alternative calculation methods revealed the same result, i.e. similar CPM responses in de novo PD and healthy controls (see Additional file 1). However, it should be noted that we did not find a significant CPM effect in the healthy controls.

Our finding is in line with previous CPM studies in medicated PD patients [22, 23, 35] reporting no differences in CPM responses between PD patients undergoing temporary withdrawal from dopaminergic medication and healthy controls. This result should be interpreted cautiously with view to the relatively small sample sizes in our and in previous studies. Nevertheless, together, these findings suggest that neither dopaminergic degeneration per se nor the dopaminergic antiparkinsonian medication have relevant effects on descending pain inhibition in PD patients, which renders it unlikely that the high prevalence of pain in PD [3] is the result of impaired descending pain modulation. However, the CPM paradigm used in our study is only one experimental way to assess the capacity for endogenous pain control and non-significant findings cannot rule 


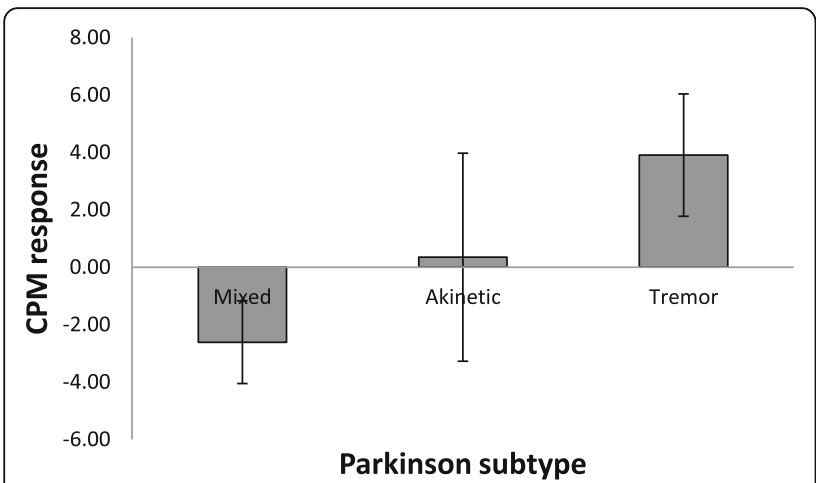

Fig. 3 Conditioned pain modulation magnitudes are shown for Parkinson subtypes. Means and standard error of means are presented. There were no significant subtype differences

out neurobiological differences in this system between PD patients and healthy controls.

It is also possible that the lack of CPM response differences between PD patients and healthy controls results from pooling PD patients across subtypes, which might vary with respect to endogenous pain control. In order to investigate whether the capacity for endogenous pain modulation varied between PD subtypes, we compared CPM responses between patients with tremor-dominant, akinetic-rigid and mixed type PD. Although differences between groups failed to reach statistical significance, it seems noteworthy that CPM responses were stronger in the tremor-dominant type than the akinetic-rigid and mixed type.

As musculoskeletal pain, the most common painful sensation in PD [17] with prevalence up to 70\% [3], is frequently associated with rigidity [17], akinetic-rigid patients might hypothetically be more likely to suffer from chronic pain than other subtypes. Given that these patients are also more impaired due to faster disease progression, higher frequency of motor fluctuations, a greater risk of cognitive dysfunction [2] and depression [47] compared to other PD subtypes, it is conceivable that the more extensive neurodegeneration in the akinetic-rigid subtype [39] also involves brain areas relevant for pain processing and modulation. Future studies on larger patient samples should therefore further explore potential subtype differences in CPM responses. As it is conceivable that CPM subtype differences might develop over the time and with disease progression, these future studies should also include PD patients with longer disease durations where potential subtype differences might be more pronounced. Moreover, in future studies with larger sample sizes, a Bayesian statistical approach would represent an appropriate way to provide more insights into potential group differences, or their absence, and PD subtypes. For that, bigger sample sizes are needed. We are aware that our subgroups are very small and that our results are not statistically significant, yet by publishing this data, we hope to draw attention to this topic of possible differences between PD subtypes.

Given the absence of significant CPM response differences between PD patients and healthy controls, abnormalities in other mechanisms involved in pain perception or pain processing in PD might be more conclusive to explain clinical pain in PD. For instance, there is first evidence for an abnormal central processing of nociceptive input [48] and abnormal brain activation in areas involved in pain processing in PD [6]. The attenuation of pain during deep brain stimulation of central brain structures, such as the globus pallidus or nucleus subthalamicus [30], also supports the hypothesis of altered central pain processing in PD. However, changes in structures such as epidermal nerve fibres / Meissner corpuscles [37] or unmyelinated nerve fibers [29] also point towards a contribution of the peripheral nervous system.

The temporal profile of pain in PD is highly variable with patients reporting pain even prior to the occurrence of motor symptoms $[45,50]$ on the one hand and pain especially dystonic pain [20] - developing under dopaminergic medication $[17,45]$ on the other hand. This indicates that pain due to dopaminergic degeneration and pain resulting from anti-parkinsonian medication might be based on different mechanisms. Furthermore, dopaminergic medication shows different effects on different types of pain in PD. For instance, levodopa increases dystonic pain [49], but improves musculoskeletal pain [25]. Future studies should therefore differentiate between different types of pain in PD (e.g. according to Ford et al. [17]) in order to investigate etiological mechanisms underlying the different types of pain.

Finally, also handedness or disease-specific body side dominancy might have influenced our results. As we used a standardized protocol, test stimuli were by defaults applied to the right arm and conditioning stimuli to the contralateral left leg. However, other researchers have used the right body side for stimulation [19] without explicitly looking at handedness or disease-specific body side dominancy. Therefore, future studies should incorporate a both-sided testing to also assess a) the influence of handedness / hand-dominancy, since nearly all of our patients were right-handed, which has been described to potentially affect experimental pain sensitivity [43] and b) the impact of the most affected body side (13 out of 17 patients were most effected on their left side) as one previous study has found an increased sensory response causing hyperalgesia in patients with predominantly leftsided PD after dopaminergic medication [22].

It should further be noted as a limitation to our study that unfortunately, after we had already obtained the data for this study, new recommendations regarding protocols for testing CPM have been published, which should be applied in future studies [55]. However, our 
study still strictly followed a previously published standardized protocol.

\section{Conclusion}

Our study, which is the first to study descending pain inhibitory mechanisms in de novo PD patients, provides further evidence against the assumption that PD is associated with a general deficit in pain regulation beyond the known age-related decline and suggests that mechanisms other than abnormalities in descending pain inhibition might explain the high pain prevalence in PD. Although differences between PD subgroups failed to reach statistical significance, stronger CPM responses in the tremordominant subtype point towards potential subgroup differences. Together, our results highlight the need for future studies in larger patient samples to elucidate the pathophysiological underpinnings of pain in PD.

\section{Additional file}

Additional file 1: Supplementary Material. (DOCX 20 kb)

\author{
Abbreviations \\ CPM: Conditioned pain modulation; CS: Conditioning stimulus; \\ HADS: Hospital Anxiety and Depression Score; PANDA: Parkinson \\ Neuropsychometric Dementia Assessment; PD: Parkinson's disease; TS: Test \\ stimulus; UPDRS: Unified Parkinson's Disease Rating Scale; VAS: Visual \\ analogue scale
}

\section{Acknowledgements}

Thanks to Katharina Wesemann and Sabrina Diedrich for their support. There were no conflicts of interest.

\section{Authors' contributions}

WG and UB designed the study. WG and OF recruited patients, acquired and analyzed data, and wrote the manuscript. CB recruited patients and analyzed data. NW, KS, and UB analyzed data and wrote the manuscript. All authors read and approved the final manuscript.

\section{Funding}

This study was supported by grants from the German Parkinson's Disease foundation (dpv), from the German Research Foundation (SFB 936/1) and from the LIIRA Grant by Pfizer. The study sponsors had no role in the study design; in the collection, analysis, and interpretation of data; in the writing of the report; and in the decision to submit the paper for publication.

\section{Availability of data and materials}

The datasets used and analyzed during the current study are available from the corresponding author on reasonable request.

\section{Ethics approval and consent to participate}

The study was conducted in accordance with the Declaration of Helsinki and approved by the local Ethics Committee of the Medical Council in Hamburg (PV3899, December 2011).

\section{Consent for publication}

All participants gave written informed consent for participation and publication of the data and were free to withdraw from the study at any time. No individual data was recorded or published.

\section{Competing interests}

The authors declare that they have no competing interests.

\section{Author details}

${ }^{1}$ Department of Neurology, University Medical Center Hamburg - Eppendorf, Martinistr. 52, 20246 Hamburg, Germany. ²Department of Neurology, University Hospital Essen, University Duisburg-Essen, Hufelandstrasse 55, 45147 Essen, Germany. ${ }^{3}$ Karolinska Institutet, 17177 Stockholm, Sweden. ${ }^{4}$ Erwin L. Hahn Institute for magnetic resonance imaging, Essen, Germany.

Received: 10 April 2019 Accepted: 6 June 2019

Published online: 26 August 2019

\section{References}

1. Arnow, B. A., Hunkeler, E. M., Blasey, C. M., Lee, J., Constantino, M. J., Fireman, B., Kraemer, H. C., Dea, R., Robinson, R., \& Hayward, C. (2006). Comorbid depression, chronic pain, and disability in primary care. Psychosomatic Medicine, 68(2), 262-268.

2. Baumann, C. R., Held, U., Valko, P. O., Wienecke, M., \& Waldvogel, D. (2014). Body side and predominant motor features at the onset of Parkinson's disease are linked to motor and nonmotor progression. Movement Disorders, 29(2), 207-213.

3. Beiske, A. G., Loge, J. H., Ronningen, A., \& Svensson, E. (2009). Pain in Parkinson's disease: Prevalence and characteristics. Pain, 141(1-2), 173-177.

4. Benarroch, E. E. (2008). Descending monoaminergic pain modulation: Bidirectional control and clinical relevance. Neurology, 71(3), 217-221.

5. Bjelland, I., Dahl, A. A., Haug, T. T., \& Neckelmann, D. (2002). The validity of the hospital anxiety and depression scale. An updated literature review. Journal of Psychosomatic Research, 52(2), 69-77.

6. Brefel-Courbon, C., Payoux, P., Thalamas, C., Ory, F., Quelven, I., Chollet, F., Montastruc, J. L., \& Rascol, O. (2005). Effect of levodopa on pain threshold in Parkinson's disease: A clinical and positron emission tomography study. Movement Disorders, 20(12), 1557-1563.

7. Brooks, D. J. (2000). Dopamine agonists: Their role in the treatment of Parkinson's disease. Journal of Neurology, Neurosurgery, and Psychiatry, 68(6), 685-689.

8. Buhmann, C., Wrobel, N., Grashorn, W., Fruendt, O., Wesemann, K., Diedrich, S., \& Bingel, U. (2017). Pain in Parkinson disease: A cross-sectional survey of its prevalence, specifics, and therapy. Journal of Neurology, 264(4), 758-769.

9. Burkey, A. R., Carstens, E., \& Jasmin, L. (1999). Dopamine reuptake inhibition in the rostral agranular insular cortex produces antinociception. The Journal of Neuroscience, 19(10), 4169-4179.

10. Castillo, R. C., Wegener, S. T., Heins, S. E., Haythornthwaite, J. A., Mackenzie, E. J., Bosse, M. J., \& Group LS. (2013). Longitudinal relationships between anxiety, depression, and pain: Results from a two-year cohort study of lower extremity trauma patients. Pain, 154(12), 2860-2866.

11. Contin, M., \& Martinelli, P. (2010). Pharmacokinetics of levodopa. Journal of Neurology, 257(Suppl 2), S253-S261.

12. Cormier, S., Piche, M., \& Rainville, P. (2013). Expectations modulate heterotopic noxious counter-stimulation analgesia. The Journal of Pain, 14(2), 114-125.

13. Edwards, R. R., Fillingim, R. B., \& Ness, T. J. (2003). Age-related differences in endogenous pain modulation: A comparison of diffuse noxious inhibitory controls in healthy older and younger adults. Pain, 101(1-2), 155-165.

14. Fahn, S. E. R. (1987). UPDRS program members. Unified Parkinsons disease rating scale. In S. MC Fahn, M. Goldstein, \& D. B. C (Eds.), Recent developments in Parkinsons disease (Vol. 2, pp. 153-163). Florham Park: Macmillan Healthcare Information.

15. Fahn, S., Oakes, D., Shoulson, I., Kieburtz, K., Rudolph, A., Lang, A., Olanow, C. W., Tanner, C., Marek, K., \& Parkinson Study, G. (2004). Levodopa and the progression of Parkinson's disease. The New England Journal of Medicine, 351(24), 2498-2508.

16. Ford, B. (2009). Parkinson disease: Pain in Parkinson disease: The hidden epidemic. Nature Reviews. Neurology, 5(5), 242-243.

17. Ford, B. (2010). Pain in Parkinson's disease. Movement Disorders, 25(Suppl 1), $\$ 98-\$ 103$.

18. Garcia-Ruiz, P. J., Chaudhuri, K. R., \& Martinez-Martin, P. (2014). Non-motor symptoms of Parkinson's disease a review...from the past. Journal of the Neurological Sciences, 338(1-2), 30-33.

19. Gerdelat-Mas, A., Simonetta-Moreau, M., Thalamas, C., Ory-Magne, F., Slaoui, T., Rascol, O., \& Brefel-Courbon, C. (2007). Levodopa raises objective pain threshold in Parkinson's disease: A RIII reflex study. Journal of Neurology, Neurosurgery, and Psychiatry, 78(10), 1140-1142.

20. Goetz, C. G., Tanner, C. M., Levy, M., Wilson, R. S., \& Garron, D. C. (1986). Pain in Parkinson's disease. Movement Disorders, 1(1), 45-49. 
21. Goffaux, P., Redmond, W. J., Rainville, P., \& Marchand, S. (2007). Descending analgesia--when the spine echoes what the brain expects. Pain, 130(1-2), 137-143.

22. Granovsky, Y., Schlesinger, I., Fadel, S., Erikh, I., Sprecher, E., \& Yarnitsky, D. (2013). Asymmetric pain processing in Parkinson's disease. European Journal of Neurology, 20(10), 1375-1382.

23. Grashorn, W., Schunke, O., Buhmann, C., Forkmann, K., Diedrich, S., Wesemann, K., \& Bingel, U. (2015). Influence of dopaminergic medication on conditioned pain modulation in Parkinson's disease patients. PLoS One, 10(8), e0135287

24. Grashorn, W., Sprenger, C., Forkmann, K., Wrobel, N., \& Bingel, U. (2013). Agedependent decline of endogenous pain control: Exploring the effect of expectation and depression. PLoS One, 8(9), e75629.

25. Ha, A. D., \& Jankovic, J. (2012). Pain in Parkinson's disease. Movement Disorders, 27(4), 485-491.

26. Hoehn, M. M., \& Yahr, M. D. (1967). Parkinsonism: Onset, progression and mortality. Neurology, 17(5), 427-442.

27. Hoehn, M. M., \& Yahr, M. D. (1967). Parkinsonism: Onset, progression, and mortality. Neurology 2001, 57(10 Suppl 3), S11-S26.

28. Kalbe, E., Calabrese, P., Kohn, N., Hilker, R., Riedel, O., Wittchen, H. U., Dodel, R, Otto, J., Ebersbach, G., \& Kessler, J. (2008). Screening for cognitive deficits in Parkinson's disease with the Parkinson neuropsychometric dementia assessment (PANDA) instrument. Parkinsonism \& Related Disorders, 14(2), 93-101.

29. Kanda, T., Tsukagoshi, H., Oda, M., Miyamoto, K., \& Tanabe, H. (1996). Changes of unmyelinated nerve fibers in sural nerve in amyotrophic lateral sclerosis, Parkinson's disease and multiple system atrophy. Acta Neuropathologica, 91(2), 145-154.

30. Kim, H. J., Jeon, B. S., \& Paek, S. H. (2011). Effect of deep brain stimulation on pain in Parkinson disease. Journal of the Neurological Sciences, 310(1-2), 251-255.

31. Lariviere, M., Goffaux, P., Marchand, S., \& Julien, N. (2007). Changes in pain perception and descending inhibitory controls start at middle age in healthy adults. The Clinical Journal of Pain, 23(6), 506-510.

32. Le Bars, D., Dickenson, A. H., \& Besson, J. M. (1979). Diffuse noxious inhibitory controls (DNIC). I. Effects on dorsal horn convergent neurones in the rat. Pain, 6(3), 283-304.

33. Millan, M. J. (2002). Descending control of pain. Progress in Neurobiology, 66(6), 355-474.

34. Moont, R., Crispel, Y., Lev, R., Pud, D., \& Yarnitsky, D. (2011). Tempora changes in cortical activation during conditioned pain modulation (CPM), a LORETA study. Pain, 152(7), 1469-1477.

35. Mylius, V., Engau, I., Teepker, M., Stiasny-Kolster, K., Schepelmann, K., Oertel, W. H., Lautenbacher, S., \& Moller, J. C. (2009). Pain sensitivity and descending inhibition of pain in Parkinson's disease. Journal of Neurology, Neurosurgery, and Psychiatry, 80(1), 24-28.

36. Nir, R. R., Yarnitsky, D., Honigman, L., \& Granot, M. (2012). Cognitive manipulation targeted at decreasing the conditioning pain perception reduces the efficacy of conditioned pain modulation. Pain, 153(1), 170-176

37. Nolano, M., Provitera, V., Estraneo, A., Selim, M. M., Caporaso, G., Stancanelli, A., Saltalamacchia, A. M., Lanzillo, B., \& Santoro, L. (2008). Sensory deficit in Parkinson's disease: Evidence of a cutaneous denervation. Brain, 131(Pt 7), 1903-1911.

38. Ossipov, M. H., Dussor, G. O., \& Porreca, F. (2010). Central modulation of pain. The Journal of Clinical Investigation, 120(11), 3779-3787.

39. Paulus, W., \& Jellinger, K. (1991). The neuropathologic basis of different clinical subgroups of Parkinson's disease. Journal of Neuropathology and Experimental Neurology, 50(6), 743-755.

40. Paulus, W., \& Trenkwalder, C. (2006). Less is more: Pathophysiology of dopaminergic-therapy-related augmentation in restless legs syndrome. Lancet Neurology, 5(10), 878-886.

41. Piche, M., Arsenault, M., \& Rainville, P. (2009). Cerebral and cerebrospinal processes underlying counterirritation analgesia. The Journal of Neuroscience, 29(45), 14236-14246.

42. Politis, M., Wu, K., Molloy, S., P, G. B., Chaudhuri, K. R., \& Piccini, P. (2010). Parkinson's disease symptoms: The patient's perspective. Movement Disorders, 25(11), 1646-1651.

43. Pud, D., Granovsky, Y., \& Yarnitsky, D. (2009). The methodology of experimentally induced diffuse noxious inhibitory control (DNIC)-like effect in humans. Pain, 144(1-2), 16-19.

44. Quittenbaum, B. H., \& Grahn, B. (2004). Quality of life and pain in Parkinson's disease: A controlled cross-sectional study. Parkinsonism \& Related Disorders, 10(3), 129-136.
45. Snider, S. R., Fahn, S., Isgreen, W. P., \& Cote, L. J. (1976). Primary sensory symptoms in parkinsonism. Neurology, 26(5), 423-429.

46. Sprenger, C., Bingel, U., \& Buchel, C. (2011). Treating pain with pain: Supraspinal mechanisms of endogenous analgesia elicited by heterotopic noxious conditioning stimulation. Pain, 152(2), 428-439.

47. Starkstein, S. E., Petracca, G., Chemerinski, E., Teson, A., Sabe, L., Merello, M., \& Leiguarda, R. (1998). Depression in classic versus akinetic-rigid Parkinson's disease. Movement Disorders, 13(1), 29-33.

48. Tinazzi, M., Recchia, S., Simonetto, S., Defazio, G., Tamburin, S., Moretto, G., Fiaschi, A., Miliucci, R., \& Valeriani, M. (2009). Hyperalgesia and laser evoked potentials alterations in hemiparkinson: Evidence for an abnormal nociceptive processing. Journal of the Neurological Sciences, 276(1-2), 153-158.

49. Tolosa, E., \& Compta, Y. (2006). Dystonia in Parkinson's disease. Journal of Neurology, 253(Suppl 7), VII7-VI13

50. Tolosa, E., Compta, Y., \& Gaig, C. (2007). The premotor phase of Parkinson's disease. Parkinsonism \& Related Disorders, 13(Suppl), S2-S7.

51. Tracey, I., \& Dickenson, A. (2012). SnapShot: Pain perception. Cell, 148(6), 1308-1308.e1302

52. Washington, L. L., Gibson, S. J., \& Helme, R. D. (2000). Age-related differences in the endogenous analgesic response to repeated cold water immersion in human volunteers. Pain, 89(1), 89-96.

53. Yarnitsky, D. (2010). Conditioned pain modulation (the diffuse noxious inhibitory control-like effect): Its relevance for acute and chronic pain states. Current Opinion in Anaesthesiology, 23(5), 611-615.

54. Yarnitsky, D. (2015). Role of endogenous pain modulation in chronic pain mechanisms and treatment. Pain, 156(Suppl 1), S24-S31.

55. Yarnitsky, D., Bouhassira, D., Drewes, A. M., Fillingim, R. B., Granot, M., Hansson, P., Landau, R., Marchand, S., Matre, D., Nilsen, K. B., Stubhaug, A., Treede, R. D., \& Wilder-Smith, O. H. (2015). Recommendations on practice of conditioned pain modulation (CPM) testing. European Journal of Pain (London, England), 19(6), 805-806.

56. Zigmond, A. S., \& Snaith, R. P. (1983). The hospital anxiety and depression scale. Acta Psychiatrica Scandinavica, 67(6), 361-370.

\section{Publisher's Note}

Springer Nature remains neutral with regard to jurisdictional claims in published maps and institutional affiliations.

Ready to submit your research? Choose BMC and benefit from:

- fast, convenient online submission

- thorough peer review by experienced researchers in your field

- rapid publication on acceptance

- support for research data, including large and complex data types

- gold Open Access which fosters wider collaboration and increased citations

- maximum visibility for your research: over $100 \mathrm{M}$ website views per year

At $\mathrm{BMC}$, research is always in progress.

Learn more biomedcentral.com/submissions 